\title{
Upregulation of retinoic acid receptor- $\beta$ reverses drug resistance in cholangiocarcinoma cells by enhancing susceptibility to apoptosis
}

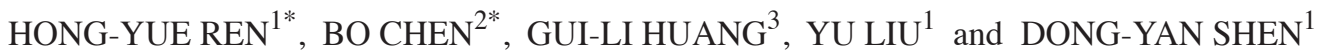 \\ ${ }^{1}$ Biobank, The First Affiliated Hospital of Xiamen University, Xiamen, Fujian 361003; ${ }^{2}$ Department of Surgical Oncology, \\ The First Affiliated Hospital of Xiamen University, Xiamen Cancer Center, Xiamen, Fujian 361003; \\ ${ }^{3}$ State Key Laboratory of Cellular Stress Biology, School of Life Sciences, \\ Xiamen University, Xiamen, Fujian 361102, P.R. China
}

Received August 11, 2015; Accepted May 26, 2016

DOI: $10.3892 / \mathrm{mmr} .2016 .5701$

\begin{abstract}
Retinoic acid receptor $\beta(\operatorname{RAR} \beta)$, a known tumor suppressor gene, is frequently silenced in numerous malignant types of tumor. Recent reports have demonstrated that loss of RAR $\beta$ expression may be responsible, in part, for the drug resistance observed in clinical trials. However, little is known about the role of RAR $\beta$ in regulating drug sensitivity in patients with cholangiocarcinoma (CCA) with a high risk of mortality and poor outcomes. In the present study, low RAR $\beta$ expression was observed in the majority of CCA tissues investigated (28/33, 84.8\%). In addition, the CCA cell line QBC939, which exhibits low RAR $\beta$ expression, was found to be significantly resistant to chemotherapeutic agents compared with SK-ChA-1, MZ-ChA-1 and Hccc9810 CCA cell lines, which exhibit high RAR $\beta$ expression. Furthermore, upregulation of RAR $\beta$ significantly enhanced the sensitivity of QBC939 cells to common chemotherapeutic agents both in vitro and in vivo. Upregulation of RAR $\beta$ was shown to increase the expression of proapoptotic genes bax, bak and bim, in addition to caspase-3 activity, and decrease the expression of antiapoptotic genes bcl-2, bcl-xL and mcl-1. As a result, CCA cells were more susceptible to caspase-dependent apoptosis. Taken together, these data suggest that RAR $\beta$ upregulation rendered CCA cells more sensitive to chemotherapeutic agents by increasing the susceptibility of cells to caspase-dependent apoptosis. These results support the hypothesis that RAR $\beta$
\end{abstract}

Correspondence to: Professor Dong-Yan Shen, Biobank, The First Affiliated Hospital of Xiamen University, 55 Zhenhai Road, Xiamen, Fujian 361003, P.R. China

E-mail: shendongyan@163.com

*Contributed equally

Key words: retinoic acid receptor $\beta$, drug resistance, caspase-3, apoptosis, cholangiocarcinoma may be an ideal chemosensitization target for the treatment of patients with drug-resistant CCA.

\section{Introduction}

Altered expression of nuclear retinoid receptors is associated with the malignant transformation of human cells (1). Retinoic acid receptor $\beta$ (RAR $\beta$ ) belongs to the RAR superfamily and is frequently silenced during epithelial cell carcinogenesis, including those of the breast, lung, esophagus, pancreas, cervix and prostate (2-7). Thus, $\operatorname{RAR} \beta$ is believed to play a role as a tumor suppressor gene in human tumorigenesis (8). Of particular note, esophageal, lung and breast cancer cell lines that do not express RAR $\beta$ have been found to be resistant to retinoid treatment $(9,10)$. This has led to the hypothesis that RAR $\beta$ expression may be responsible, in part, for mediating the efficacy of chemopreventative agents in clinical trials, such as inhibitors of the epidermal growth factor receptor and $\beta$-cryptoxanthin. An increasing number of studies suggest that restoration of RAR $\beta$ expression in tumor cells may enhance their response to chemotherapeutic agents (11).

Cholangiocarcinoma (CCA) is the second most common primary liver malignancy, which is highly resistant to available chemotherapeutic agents and confers a 5-year relative survival rate of less than $5 \%$ (12). Surgery is the only curative treatment for CCA, however only a very small percentage of tumors are resectable. This is primarily due to the majority of patients presenting with late-stage disease, such as local advanced or metastatic disease (13). Therefore, the identification of new and effective therapies against CCA is critical. To date, clinical strategies to enhance drug sensitivity have included efforts that aim to initiate apoptotic signaling pathways, activate specific tumor suppressors and determine effective combination regimens (14). However, expression of the RAR $\beta$ tumor suppressor in CCA, together with its role in regulating sensitivity to chemotherapeutic agents, are currently unknown.

In the present study, the expression of RAR $\beta$ in CCA tissues was investigated and the potential mechanisms by which RAR $\beta$ confers sensitivity to chemotherapeutic agents within CCA cells in vitro and in vivo was explored. 


\section{Materials and methods}

Reagents. 5-fluorouracil (5-FU), vincristine sulfate (VCR), cisplatin (CDDP), mitomycin C (MMC), 3-(4,5-dimethylthiazol-2-yl)-2,5-diphenyltetrazolium bromide (MTT), caspase-3 inhibitor (z-VAD-fmk), G418, BMS453 and $\mathrm{CoCl} 2$ were all purchased from Sigma-Aldrich (St. Louis, MO, USA). The RPMI-1640 and fetal bovine serum (FBS) were all purchased from Gibco BRL (Grand Island, NY). The $100 \mathrm{U} / \mathrm{ml}$ penicillin and bicinchoninic acid protein assay reagent were purchased from Wuhan Boster (Wuhan, China). Glyceraldehyde 3-phosphate dehydrogenase (GAPDH; ab9485) and RAR $\beta$ (ab198557) antibodies were purchased from Abcam (Cambridge, UK). The annexin V-fluorescein isothiocyanate (FITC)/propidium iodide (PI) double staining apoptosis detection kit and TdT-mediated dUTP nick end labeling (TUNEL) kit were obtained from Roche Diagnostics (Basel, Switzerland). Lipofectamine 2000 was purchased from Invitrogen (Thermo Fisher Scientific, Inc., Waltham, MA., USA). The Caspase-3 assay kit was from R\&D Systems, Inc. (Minneapolis, MN, USA). All of other chemicals and reagents were the highest quality and purchased from standard commercial sources.

Patients and tumor specimens. Analysis of RAR $\beta$ expression in clinical CCA tissues was performed in agreement with the ethical guidelines of the 1975 Declaration of Helsinki, and was approved by the Institute Research Ethics Committee at The First Affiliated Hospital of Xiamen University. Between 2009-2013, 33 CCA samples were collected from patients without metastatic disease that had not received pre-operative treatment. Tumor tissues were fixed in $10 \%$ formalin and then paraffin-embedded for immunohistochemical (IHC) analysis and routine histological characterization.

Cell culture and stable transfection. The human CCA cell line QBC939 was a kind gift from Professor Shu-Guang Wang from Southwest Hospital, the Third Military Medical University (Chongqing, China). The CCA cell lines Sk-ChA-1, MZ-ChA-1 and Hccc9810 were a kind gift from Professor Chun-Dong Yu laboratory of Xiamen University (Xiamen, China). HIBEpiC human intrahepatic biliary epithelial cells were purchased from Cell Bank of the Type Culture Collection of Chinese Academy of Sciences (Shanghai, China). All four human CCA cell lines and HIBEpiC cells were all cultured in RPMI-1640 supplemented with 10\% FBS and $100 \mathrm{U} / \mathrm{ml}$ penicillin in a humidified chamber at $37^{\circ} \mathrm{C}$ in $5 \% \mathrm{CO}_{2}$. RAR $\beta$ cDNA was cloned into the expression vector $\mathrm{pBoBi}$ as described previously (15). The RAR $\beta$ expression vector $(4 \mu \mathrm{g})$ was stably transfected into QBC939 cells $\left(1 \times 10^{6}\right)$ to produce $\mathrm{pBoBi}-\mathrm{RAR} \beta$ using $10 \mu \mathrm{l}$ Lipofectamine 2000 , according to the manufacturer's instructions. Positive selection of stable transfectants was achieved by supplementing complete medium with $400 \mathrm{mg} / \mathrm{ml}$ of G418. Stable control vector QBC939 transfectants are defined as pBoBi-Ctrl from herein.

Cell proliferation assay. To determine cell viability in response to treatment, an MTT assay was used. Briefly, QBC939 cells were seeded onto 96 -well culture plates at a density of $5 \times 10^{3}$ cells/well and grown in complete RPMI-1640 culture medium. Following overnight incubation, cells were treated with a variety of agents $(60 \mu \mathrm{M})$ including 5-FU, CDDP, VCR and MMC for $48 \mathrm{~h}$. MTT $(5 \mathrm{mg} / \mathrm{ml})$ was added to each well and incubated at $37^{\circ} \mathrm{C}$ for $4 \mathrm{~h}$, before the resulting formazan crystals were dissolved in dimethyl sulfoxide. Absorbance was read at $490 \mathrm{~nm}$ using a microplate reader (Model 680; Bio-Rad Laboratories, Inc., Hercules, CA, USA).

Hypoxic growth conditions. Cells were cultured in a $35 \mathrm{~mm}$ dish until 70-80\% confluence was reached. They were then transferred to a hypoxic incubator with a humidified atmosphere that was flushed with $\leq 0.1 \% \mathrm{O}_{2}, 5 \% \mathrm{CO}_{2}$ and $95 \% \mathrm{~N}_{2}$, followed by incubation at $37^{\circ} \mathrm{C}$ for $12 \mathrm{~h}$.

Serum starvation. Cells were seeded onto 6-well culture plates at a density of $2 \times 10^{5}$ cells/well and grown in complete RPMI-1640 culture medium overnight. Then the medium was removed and washed with PBS, and added the RPMI 1640 medium without serum. The duration of serum starvation was determined according to the experimental requirements.

Tumor xenograft study. In vivo drug sensitivity experiments were divided into two groups: RAR $\beta$ (pBoBi-RAR $\beta$ ) and control (pBoBi-Ctrl). Female specific pathogen-free BALB/c nude mice (Shanghai Laboratory Animal Center, Shanghai, China) (age, 4-5 weeks) were injected subcutaneously with $100 \mu \mathrm{l}$ cells $\left(2 \times 10^{6}\right)$. Mice were treated with $70 \mathrm{mg} / \mathrm{kg} / \mathrm{d} 5-\mathrm{FU}$ at day 8 post-transplantation. Tumor volumes were determined using the following formula: $\left(\mathrm{AxB}^{2}\right) / 2$, where $\mathrm{A}$ is the largest diameter and $\mathrm{B}$ is the perpendicular diameter. After 35 days from injection, mice were sacrificed by inhalation of carbon dioxide. All animal procedures were approved by the Animal Care and Use Committee of the First Affiliated Hospital of Xiamen University.

Gene and protein expression analyses. mRNA expression levels were determined by reverse transcription-quantitative polymerase chain reaction (RT-qPCR). Protein expression was measured using a western blotting assay. Both analyses were performed as described previously (15).

Immunohistochemical (IHC) staining. IHC was performed to detect RAR $\beta$ expression in CCA tissues as described previously (16). Human CCA tissue sections were immunostained with an antibody against $\operatorname{RAR} \beta$ (1:100). IHC scoring was conducted by two independent pathologists. The number of positively-stained cells per 1,000 cells in randomly selected fields was recorded, and the mean number from a minimum of five slides was calculated. Staining intensity was categorized as low $(<50 \%)$ or high $(>50 \%)$.

Analysis of apoptosis. Apoptosis was evaluated using an annexin V-FITC/PI double-staining assay and a TUNEL assay. The annexin V-FITC/PI double-staining assay was performed according to the manufacturer's instructions (Roche Diagnostics) and analyzed using a flow cytometer (FACSCabilur; BD Biosciences, Franklin Lakes, CA, USA). TUNEL staining was performed using an in situ cell death detection kit according to the manufacturer's instructions (Roche Diagnostics). 
Analysis of caspase-3 activity. Caspase-3 activity was determined using the caspase-3 assay kit (R\&D Systems Inc., Minneapolis, MN, USA) according to the manufacturer's instructions. Briefly, cells were lysed and centrifuged at $10,000 \mathrm{x} \mathrm{g}$ for $20 \mathrm{~min}$ at $4^{\circ} \mathrm{C}$ to obtain supernatants. The protein concentration of sample supernatants was determined using a bicinchoninic acid protein assay reagent before they were added to a dithiothreitol and caspase- 3 substrate reaction mixture and incubated for $2 \mathrm{~h}$ at $37^{\circ} \mathrm{C}$. Absorbance was measured at $405 \mathrm{~nm}$ using a microplate reader (Model 680; Bio-Rad Laboratories, Inc.).

Statistical analysis. Data are presented as the mean \pm standard error from a minimum of three independent experiments. Student's $t$-test or one-way analysis of variance was conducted using the SPSS 13.0 software package (SPSS, Inc., Chicago, IL, USA). Dunn test was used if there was statistical significance after one-way analysis. $\mathrm{P}<0.05$ was considered to indicate a statistically significant difference.

\section{Results}

Low expression of $R A R \beta$ is partially responsible for the drug resistance of $C C A$. IHC was performed to investigate RAR $\beta$ protein expression in a set of 33 paraffin-embedded human CCA tissues. A total of 28/33 (84.8\%) tissues were found to exhibit low RAR $\beta$ protein expression (Fig. 1A), only 5 tissues exhibited high RAR $\beta$ protein expression (Fig. 1B). In addition, RAR $\beta$ mRNA and protein expression levels in four CCA cell lines (QBC939, SK-ChA-1, MZ-ChA-1 and Hccc9810 cells) were found to be significantly lower than the HBd Epi normal bile duct epithelial cell line (Fig. 2A). RAR $\beta$ mRNA and protein levels were markedly reduced within QBC939 cells. Notably, the proportion of surviving QBC939 cells following exposure to three commonly used chemotherapeutic agents including 5-FU, VCR and MMC, was found to be significantly higher compared with the remaining three CCA cell lines (Fig. 2B). These results give rise to the hypothesis that the observed resistance of CCA cells to common therapeutic agents, may be associated with silenced RAR $\beta$ expression.

Upregulation of RAR $\beta$ enhances the sensitivity of CCA cells to chemotherapeutic agents in vitro. In order to investigate the role of RAR $\beta$ in the therapeutic response of CCA cells, RAR $\beta$ expression vectors were transfected into QBC939 cells prior to treatment with chemotherapeutic agents. As shown in Fig. 3A, an increase in RAR $\beta$ mRNA and protein levels was observed within stably RAR $\beta$-transfected cells (pBoBi-QBC939) compared with control (pBoBi-Ctrl) cells. Cell survival analysis results indicated that the sensitivity of pBoBi-QBC939 cells to common chemotherapeutic agents, including 5-FU, CDDP, VCR and MMC, was significantly enhanced following RAR $\beta$ upregulation compared with pBoBi-Ctrl cells (Fig. 3B). Furthermore, the proportion of apoptotic pBoBi-QBC939 cells generated in response to 5-FU, CDDP, VCR and MMC, was found to be significantly higher than $\mathrm{pBoBi}-\mathrm{C}$ trl cells (Fig. $3 \mathrm{C}$ ). These in vitro findings suggest that upregulation of RAR $\beta$ enhances the sensitivity of CCA cells to chemotherapeutic agents.

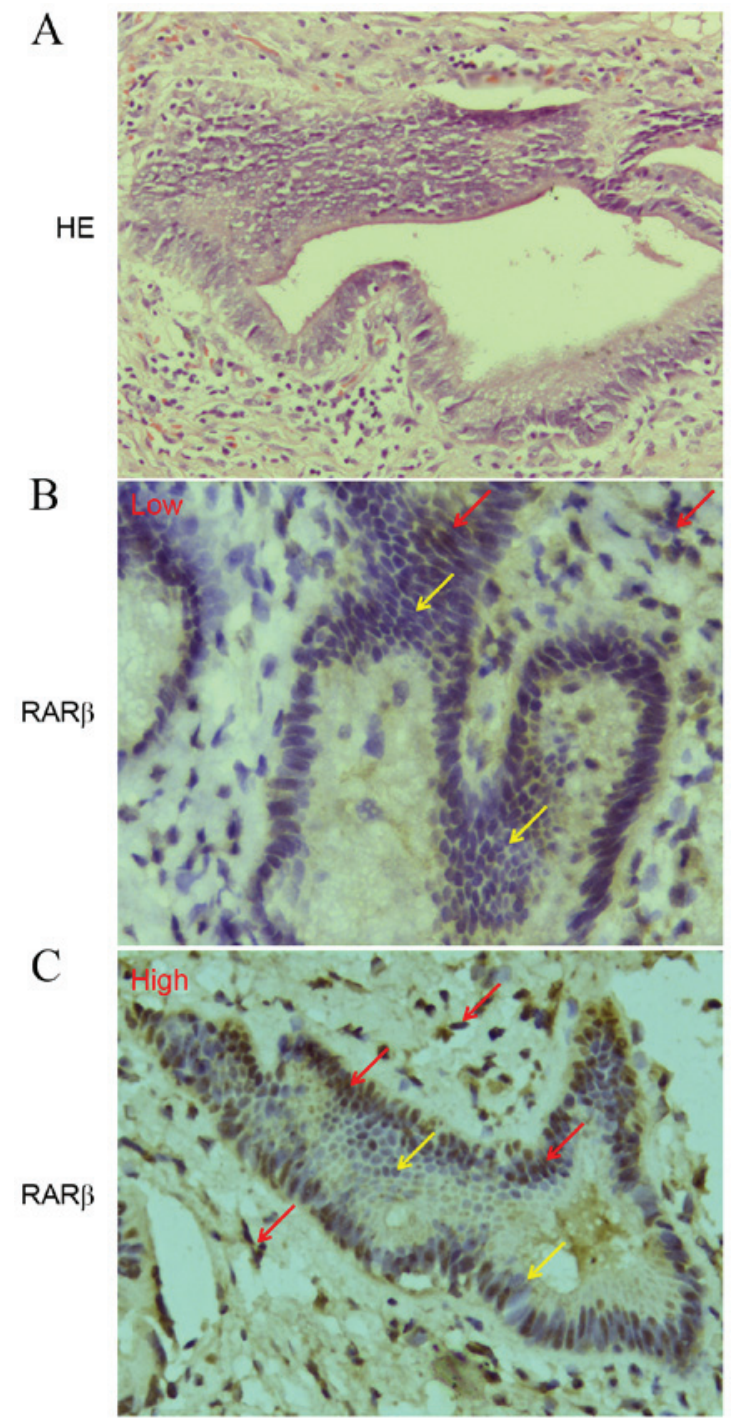

Figure 1. Expression of RAR $\beta$ protein detected by IHC in human CCA tissues. (A) HE staining. (B and C) Expression of RAR $\beta$ (magnification, $x 400$ ). Yellow arrows indicate negative RAR $\beta$ expression and red arrows indicate positive expression. The staining intensities of RAR $\beta$ were categorized as low and high expression in $33 \mathrm{CCA}$ tissues. $\mathrm{RAR} \beta$, retinoic acid receptor- $\beta$; IHC, immunohistochemical staining; CCA, cholangiocarcinoma; HE, hematoxylin-eosin.

Upregulation of RAR $\beta$ increases the cytotoxic effect of 5-FU on xenografted CCA tumors. 5-FU is a common chemotherapeutic agent used for the treatment of hepatobiliary tumors. Therefore, this agent was selected to investigate the contribution of RAR $\beta$ upregulation in the sensitivity to chemotherapeutic treatment in the xenografted QBC939 CCA tumors. As shown in Fig. 4A, the rate of tumor growth inhibition in xenografted pBoBi-RAR $\beta$ tumors following treatment with 5-FU, was significantly higher than in control pBoBi-Ctrl tumors. A significant difference in tumor growth inhibition was apparent from day 20 and increased further during late tumor growth (Fig. 4B). Moreover, analysis of apoptosis in xenografted CCA tumor tissues using the TUNEL assay was consistent with the results of the in vitro assays, and indicated that the number of apoptotic cells induced by 5 -FU in xenografted pBoBi-RAR $\beta$ tumors was significantly higher than control pBoBi-Ctrl tumors $(\mathrm{P}=0.0035$; Fig. $4 \mathrm{C})$. These in vivo findings provide 
A
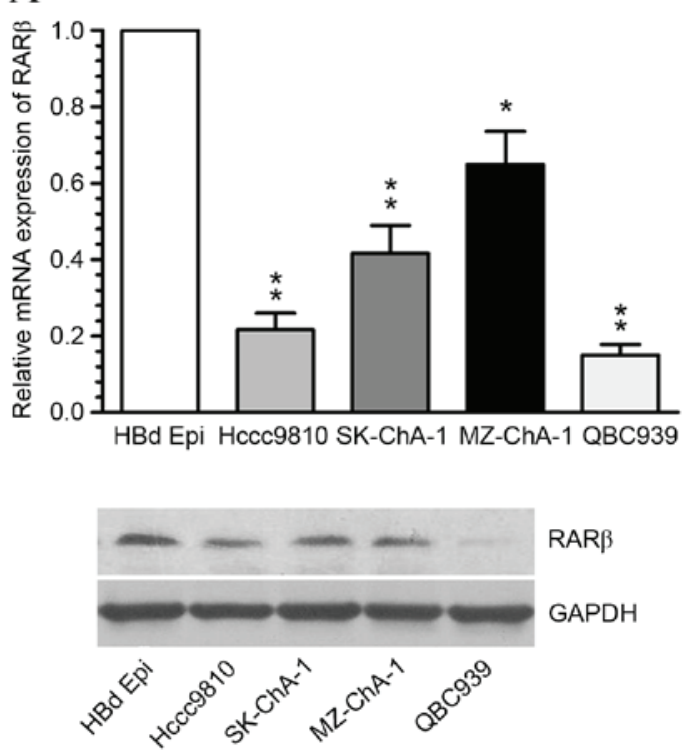

B

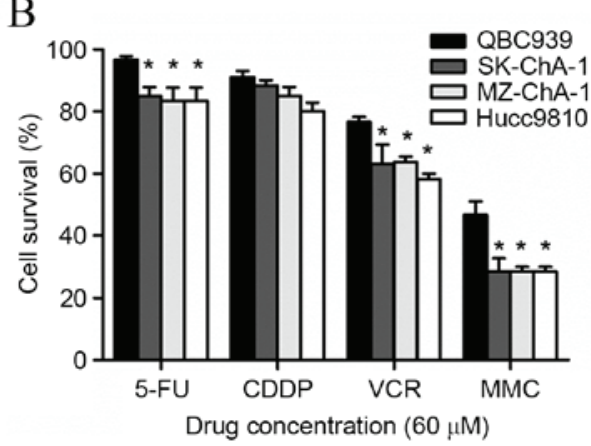

Figure 2. Expression of RAR $\beta$ and drug sensitivity in human CCA cell lines. (A) Relative mRNA expression levels of RAR $\beta$ in the normal bile duct cells and 4 CCA cell lines, as detected by reverse transcription-quantitative polymerase chain reaction. ${ }^{*} \mathrm{P}<0.05,{ }^{* *} \mathrm{P}<0.001$ vs. HBd Epi cells. Protein expression detected by western blotting analysis. (B) Drug sensitivities of four CCA cell lines in response to 5-FU, CDDP, VCR and MMC chemotherapeutic agents as determined using the 3-(4,5-dimethylthiazol-2-yl)-2,5-diphenyltetrazolium bromide assay. ${ }^{*} \mathrm{P}<0.05,{ }^{* *} \mathrm{P}<0.001$ vs. QBC939 cells. RAR $\beta$, retinoic acid receptor- $\beta$; $\mathrm{CCA}$, cholangiocarcinoma; 5 -FU, 5 -fluorouracil; CDDP, cisplatin; VCR, vincristine; MMC, mitomycin C.

additional evidence that RAR $\beta$ upregulation enhances the sensitivity of CCA cells to chemotherapeutic agents.

High RAR $\beta$ expression renders CCA cells more susceptible to caspase-dependent apoptosis. Taking the above results into consideration, the observed increase in the sensitivity of CCA cells to chemotherapeutic agents following RAR $\beta$ upregulation may render CCA cells more susceptible to apoptosis. Hence, the expression levels of genes associated with apoptosis following $\operatorname{RAR} \beta$ upregulation were investigated. Results from RT-qPCR assays showed that the expression of proapoptotic genes, including bax, bak and bim, were increased $>4$ fold in pBoBi-RAR $\beta$ QBC939 cells, while the expression of antiapoptotic genes, including bcl-2, bcl-xL and mcl-1, were all significantly decreased compared with pBoBi-Ctrl cells (Fig. 5A). To investigate these findings further, the association between RAR $\beta$ expression and caspase- 3 activity was explored. Treatment of pBoBi-RAR $\beta$ cells with the RAR $\beta$-activator, BMS453,
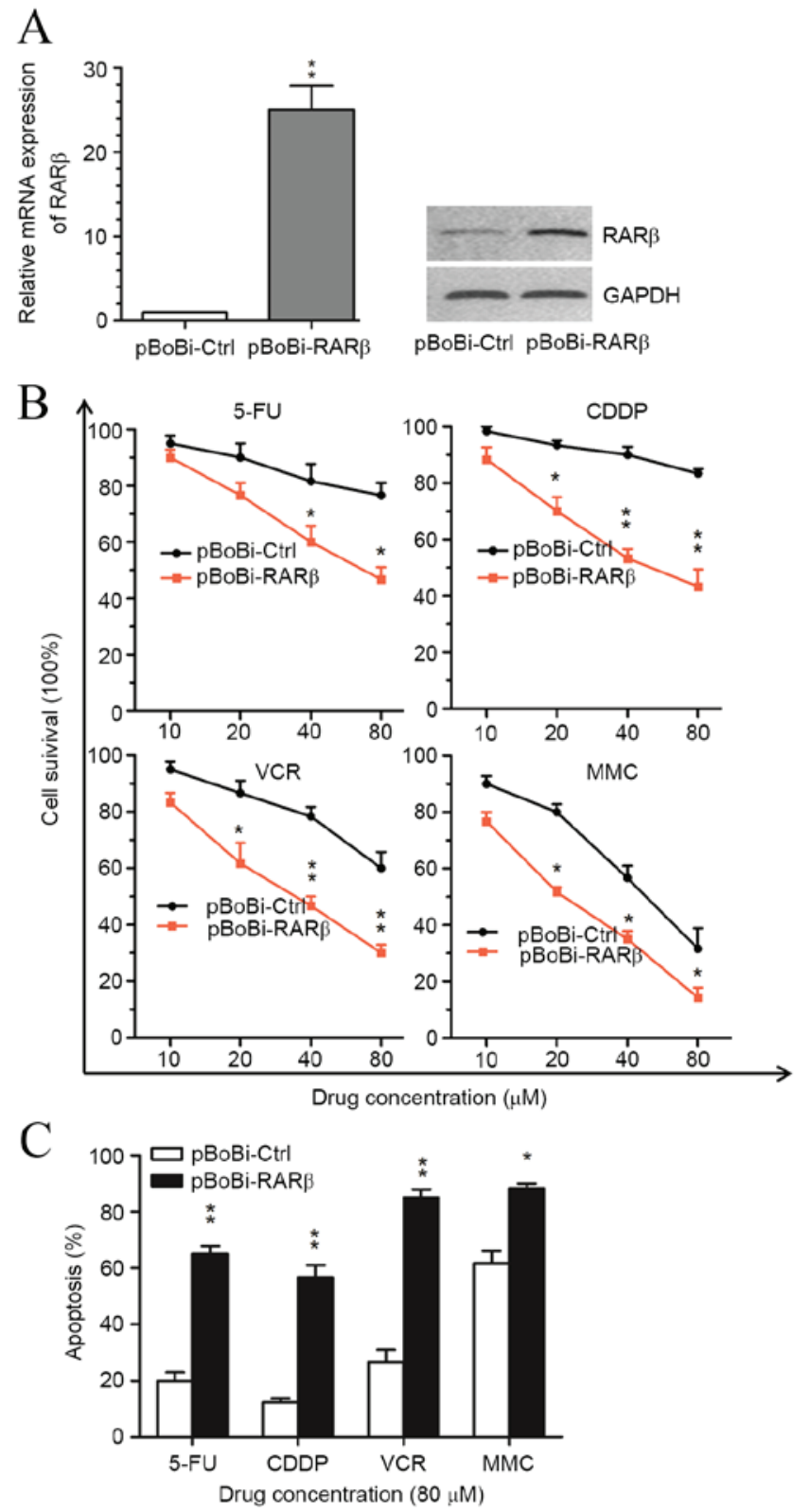

Figure 3. Sensitivity of QBC939 cells to chemotherapeutic agents after RAR $\beta$ upregulation. Expression of (A) RAR $\beta$ mRNA and protein in QBC939 cells following stable transfection with RAR $\beta$ (pBoBi-RAR $\beta$ ) or control (pBoBi-Ctrl) expression vectors. (B) Cell survival analysis of pBoBi-Ctrl and $\mathrm{pBoBi}-\mathrm{RAR} \beta$ cells following treatment with 5-FU, CDDP, VCR and MMC chemotherapeutic agents for $48 \mathrm{~h} .{ }^{*} \mathrm{P}<0.05,{ }^{* *} \mathrm{P}<0.001$ vs. pBoBi-Ctrl. (C) The proportion of apoptotic pBoBi-Ctrl and pBoBi-RAR $\beta$ QBC939 cells induced by each chemotherapeutic agent. ${ }^{*} \mathrm{P}<0.05,{ }^{* *} \mathrm{P}<0.01$ vs. pBoBi-Ctrl. RAR $\beta$, retinoic acid receptor- $\beta$; 5 -FU, 5 -fluorouracil; CDDP, cisplatin; VCR, vincristine; $\mathrm{MMC}$, mitomycin $\mathrm{C}$.

resulted in a 3.5-fold increase in caspase-3 activity compared with pBoBi-Ctrl cells, which exhibited a 1.8-fold increase. The observed increase in caspase-3 activity was further enhanced when cells were cultured under hypoxic conditions or following serum starvation. By contrast, the activity of caspase- 3 was significantly decreased in both pBoBi-RAR $\beta$ and $\mathrm{pBoBi}-\mathrm{Ctrl}$ cells after treatment with the caspase-3 inhibitor z-VAD-fmk, even when cells were cultured under hypoxic conditions or following serum starvation (Fig. 5B). Furthermore, apoptosis induced by 5-FU, hypoxia or serum 

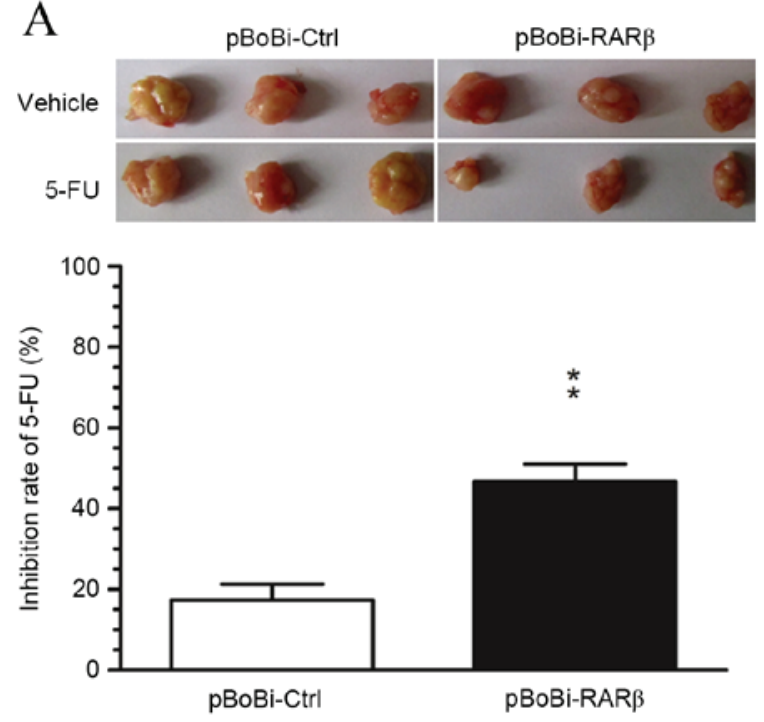

B

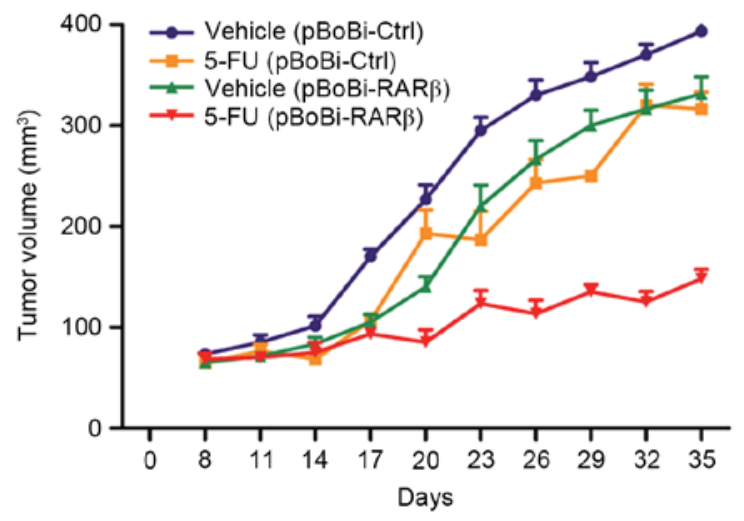

$\mathrm{C}$

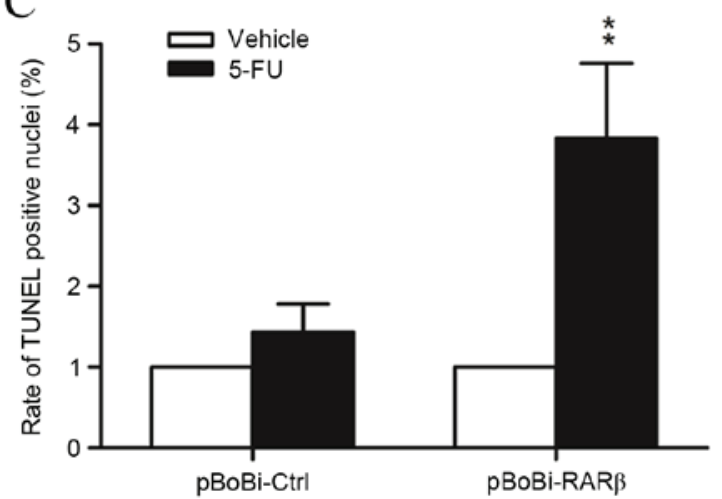

Figure 4. RAR $\beta$ upregulation contributes to the cytotoxic effect of 5-FU in vivo. (A) Tumor images and quantitative analysis of tumor weight show the rate of growth inhibition of CCA xenograft tumors following 5-FU treatment. ${ }^{*} \mathrm{P}<0.05,{ }^{* *} \mathrm{P}<0.001$ vs. pBoBi-Ctrl. (B) Tumor volume $\left(\mathrm{mm}^{3}\right)$ of CCA xenograft tumors at 8-35 days post-injection. Tumor latency was considered to be the time taken for the tumor to grow to a size of $80 \mathrm{~mm}^{3}$. (C) The number of apoptotic cells in CCA xenograft tumors was detected using the TUNEL assay. ${ }^{*} \mathrm{P}<0.05,{ }^{* *} \mathrm{P}<0.01$ vs. pBoBi-Ctrl. RAR $\beta$, retinoic acid receptor- $\beta$; CCA, cholangiocarcinoma; 5 -FU, 5 -fluorouracil; TUNEL, TdT-mediated dUTP nick end labeling.

starvation was enhanced upon upregulation or activation of RAR $\beta$ (Fig. 5C). These results indicate that the susceptibility
A

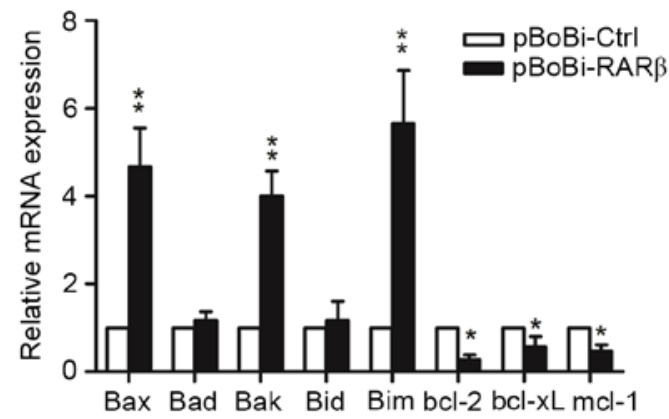

$\mathrm{B}$

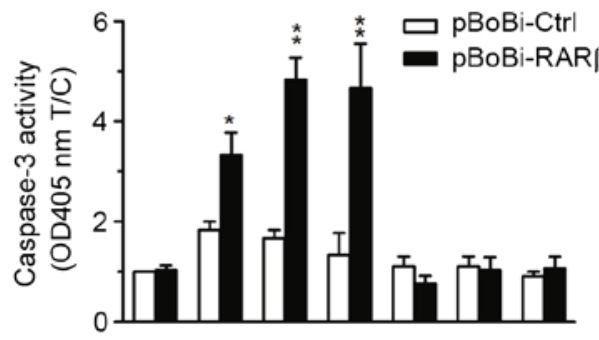

$\begin{array}{rccccccc}\text { BMS453: } & - & + & + & + & + & + & + \\ \text { Z-VAD-fink: } & - & - & - & - & + & + & + \\ \text { Hypoxia: } & - & - & + & - & - & + & - \\ \text { Serum starvation: } & - & - & - & + & - & - & +\end{array}$

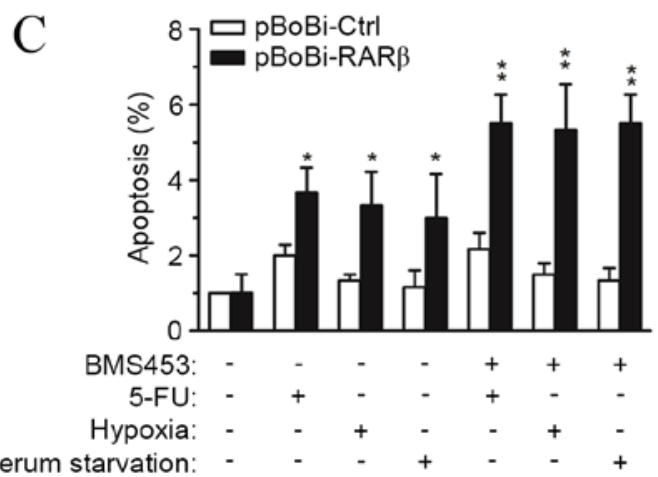

Figure 5. Efficacy of RAR $\beta$ upregulation on cholangiocarcinoma apoptosis induction. (A) Analysis of apoptosis-associated gene expression by reverse transcription-quantitative polymerase chain reaction in $\mathrm{pBoBi}-\mathrm{RAR} \beta$ and pBoBi-Ctrl QBC939 cells. ${ }^{*} \mathrm{P}<0.05,{ }^{* *} \mathrm{P}<0.001$ vs. pBoBi-Ctrl. (B) Caspase-3 activity within $\mathrm{pBoBi}-\mathrm{RAR} \beta$ and $\mathrm{pBoBi}-\mathrm{Ctrl} \mathrm{QBC} 939$ cells following treatment with the RAR $\beta$-activator BMS453 $(10 \mu \mathrm{M})$, the caspase-3 inhibitor Z-VAD-fmk $(20 \mu \mathrm{M})$, or following hypoxia or serum starvation for $6 \mathrm{~h} .{ }^{*} \mathrm{P}<0.05,{ }^{* *} \mathrm{P}<0.001$ vs. pBoBi-Ctrl. (C) The proportion of apoptotic pBoBi-RAR $\beta$ and pBoBi-Ctrl QBC939 cells following treatment with BMS453 $(10 \mu \mathrm{M}), 5-\mathrm{FU}(60 \mu \mathrm{M})$ or following hypoxia or serum starvation for 12 h. ${ }^{*} \mathrm{P}<0.05,{ }^{* *} \mathrm{P}<0.01$ vs. pBoBi-Ctrl. RAR $\beta$, retinoic acid receptor- $\beta$; 5-FU, 5-fluorouracil.

of CCA cells to caspase-dependent apoptosis may be enhanced by the upregulation of RAR $\beta$.

\section{Discussion}

The results of the present study indicate the role of RAR $\beta$ upregulation on the sensitivity of CCA cells to chemotherapeutic agents. Of note, RAR $\beta$ upregulation was found to increase the sensitivity of CCA cells and tumors to various chemotherapeutic agents currently used for the treatment of CCA. In addition, RAR $\beta$ upregulation was observed to be associated with an increase in the $\mathrm{Bax} / \mathrm{Bcl}-2$ ratio and caspase- 3 
activity, and rendered CCA cells and xenograft tumors more susceptible to apoptosis. These findings provide evidence to suggest that the upregulation of RAR $\beta$ may represent a beneficial adjunct therapeutic strategy for the treatment of CCA.

Previous studies have shown that loss of RAR $\beta$ is a common event in the development of malignant tumors, and the induction of RAR $\beta$ expression suppresses cancer development $(2-7,17)$. Increased expression of RAR $\beta$ has been found to correlate with the clinical response to various retinoids $(1,18)$; however, the molecular mechanism of $\operatorname{RAR} \beta$ in regulating sensitivity to chemotherapeutic agents is little known. A major problem in the treatment of human cancer is the lack of response of many tumors to chemotherapeutic agents. Following exposure to anti-cancer agents, tumor cells can alter the expression profile of a specific set of genes to activate and/or suppress signaling networks, which de-sensitizes cells to drug-induced death signals and confers cell survival and chemoresistance $(19,20)$.

Similar to other types of human carcinoma, the results of the present study indicate that CCA, a chemoresistant bile duct carcinoma with a poor prognosis (21), demonstrates loss of RAR $\beta$ expression. Upregulation of RAR $\beta$ within the drug-resistant CCA cell line QBC939, which exhibits loss of RAR $\beta$ expression, was observed to increase the susceptibility of cells to apoptosis induced by chemotherapeutic agents in vitro and in vivo. Hypoxia and nutrient deficiency are common adverse microenvironments encountered by tumor cells. Based on the presence of specific intracellular signaling pathways, tumor cells can either become more aggressive or undergo apoptosis in response to these microenvironmental stress conditions. Differentially expressed genes, including tumor suppressor genes and oncogenes, are involved in the processes of adaptation. Once upregulated, tumor suppressor genes can initiate apoptosis (22). Consistent with these observations, upregulation of RAR $\beta$ in the present study was found to induce CCA cells to undergo apoptosis in response to hypoxic and starvation conditions.

It is possible that $\operatorname{RAR} \beta$ upregulation may initiate the process of apoptosis. It is known that the Bcl-2 family of proteins regulates the apoptotic pathway by balancing the expression of proapoptotic (Bax) and antiapoptotic ( $\mathrm{Bcl}-2)$ factors. In this regard, the $\mathrm{Bax} / \mathrm{Bcl}-2$ ratio is suggested to be a useful predictor of apoptotic cell death (23). In the present study, RAR $\beta$ upregulation was found to induce changes in the expression of genes belonging to the $\mathrm{Bcl}-2$ family, which resulted in an increase in the Bax/Bcl-2 ratio. Caspase-3 functions as an executor of apoptosis by activating DNA fragmentation (24). During the apoptotic processes, cytochrome $\mathrm{c}$ is released from the mitochondria and into the cytosol. Cytoplasmic cytochrome $\mathrm{c}$ then activates caspase-9, which in turn triggers the activation of caspase- 3 and leads to cell death. Increased caspase-3 activity is associated with an increase in the $\mathrm{Bax} / \mathrm{Bcl}-2$ ratio (25). Despite the fact that the expression of cytochrome $\mathrm{c}$ and caspase-9 was not investigated in the present study, apoptosis induced by RAR $\beta$ upregulation was observed to be associated with the activation of caspase-3, which led to an increase in the Bax/Bcl-2 ratio.

In conclusion, the data presented in the present study demonstrate a clear functional role for RAR $\beta$ in sensitizing
CCA cells to chemotherapy-induced cell death. Taken in conjunction with previous studies, it is proposed that the use of adjunct therapies that confer RAR $\beta$ upregulation should be explored for the treatment of chemoresistant tumors, such as CCA, with the aim of overcoming drug resistance and/or increasing the susceptibility of tumor cells to initiate intrinsic cell death pathways.

\section{Acknowledgements}

The present study was supported by grants from National Nature Science Foundation of China (grant nos. 81572394 and 81201892) and the Project from Science and Technology Bureau of Xiamen, China (grant no. 3502Z20144002).

\section{References}

1. Bialešová L, Brtko J, Lenko V and Macejová D: Nuclear receptors-target molecules for isoflavones in cancer chemoprevention. Gen Physiol Biophys 32: 467-478, 2013.

2. Brtko J: Role of retinoids and their cognate nuclear receptors in breast cancer chemoprevention. Cent Eur J Public Health 15: 3-6, 2007.

3. Hua F, Fang N, Li X, Zhu S, Zhang W and Gu J: A meta-analysis of the relationship between RAR $\beta$ gene promoter methylation and non-small cell lung cancer. PloS One 9: e96163, 2014

4. Liu Z, Zhang L, Ding F, Li J, Guo M, Li W, Wang Y, Yu Z, Zhan Q, Wu M and Liu Z: 5-Aza-2'-deoxycytidine induces retinoic acid receptor-beta(2) demethylation and growth inhibition in esophageal squamous carcinoma cells. Cancer Lett 230: 271-283, 2005.

5. Pérez RJ, Benoit YD and Gudas LJ: Deletion of retinoic acid receptor $\beta(\operatorname{RAR} \beta)$ impairs pancreatic endocrine differentiation. Exp Cell Res 319: 2196-2204, 2013.

6. Terra AP, Murta EF, Maluf PJ, Caballero OL, Brait M and Adad SJ: Aberrant promoter methylation can be useful as a marker of recurrent disease in patients with cervical intraepithelial neoplasia grade III. Tumori 93: 572-579, 2007.

7. Moison C, Assemat F, Daunay A, Tost J, Guieysse-Peugeot AL and Arimondo PB: Synergistic chromatin repression of the tumor suppressor gene RARB in human prostate cancers. Epigenetics 9: 477-482, 2014.

8. Alvarez S, Germain P, Alvarez R, Rodríguez-Barrios F, Gronemeyer $\mathrm{H}$ and de Lera AR: Structure, function and modulation of retinoic acid receptor beta, a tumor suppressor. Int $\mathrm{J}$ Biochem Cell Biol 39: 1406-1415, 2007.

9. Peng X, Green A, Shilkaitis A, Zhu Y, Bratescu L and Christov K: Early in vitro passages of breast cancer cells are differentially susceptible to retinoids and differentially express RAR $\beta$ isoforms. Int J Oncol 39: 577-583, 2011.

10. Fernández-Martínez AB and Lucio Cazaña FJ: Epidermal growth factor receptor transactivation by intracellular prostaglandin E2-activated prostaglandin E2 receptors. Role in retinoic acid receptor- $\beta$ up-regulation. Biochim Biophys Acta 1833: 2029-2038, 2013.

11. Bu P and Wan YJ: Fenretinide-induced apoptosis of Huh-7 hepatocellular carcinoma is retinoic acid receptor beta dependent. BMC Cancer 7: 236, 2007.

12. Zhang W and Yan LN: Perihilar cholangiocarcinoma: Current therapy. World J Gastrointest Pathophysiol 5: 344-354, 2014.

13. Unno M: Review of surgical treatment of perihilar cholangiocarcinoma: Proper patient selection for combined vascular resection and reconstruction. Nihon Geka Gakkai zasshi 115: 181-184, 2014 (In Japanese).

14. Demonceau J, Ruppar T, Kristanto P, Hughes DA, Fargher E, Kardas P, De Geest S, Dobbels F, Lewek P, Urquhart J, et al: Identification and assessment of adherence-enhancing interventions in studies assessing medication adherence through electronically compiled drug dosing histories: A systematic literature review and meta-analysis. Drugs 73: 545-562, 2013.

15. Huang GL, Luo Q, Rui G, Zhang W, Zhang QY, Chen QX and Shen DY: Oncogenic activity of retinoic acid receptor $\gamma$ is exhibited through activation of the $\mathrm{Akt} / \mathrm{NF}-\kappa \mathrm{B}$ and $\mathrm{Wnt} / \beta$-catenin pathways in cholangiocarcinoma. Mol Cell Biol 33: 3416-3425, 2013. 
16. Shen DY, Fang ZX, You P, Liu PG, Wang F, Huang CL, Yao XB Chen ZX and Zhang ZY: Clinical significance and expression of cyclin kinase subunits 1 and 2 in hepatocellular carcinoma. Liver Int 30: 119-125, 2010.

17. Xu XC: Tumor-suppressive activity of retinoic acid receptor-beta in cancer. Cancer Lett 253: 14-24, 2007.

18. Wan H, Oridate N, Lotan D, Hong WK and Lotan R: Overexpression of retinoic acid receptor beta in head and neck squamous cell carcinoma cells increases their sensitivity to retinoid-induced suppression of squamous differentiation by retinoids. Cancer Res 59: 3518-3526, 1999.

19. Itamochi H, Kigawa J and Terakawa N: Mechanisms of chemoresistance and poor prognosis in ovarian clear cell carcinoma. Cancer Sci 99: 653-658, 2008.

20. Sui X, Kong N, Ye L, Han W, Zhou J, Zhang Q, He C and Pan H: p38 and JNK MAPK pathways control the balance of apoptosis and autophagy in response to chemotherapeutic agents. Cancer Lett 344: 174-179, 2014
21. Morita SY and Terada T: Molecular mechanisms for biliary phospholipid and drug efflux mediated by ABCB4 and bile salts. Biomed Res Int 2014: 954781, 2014.

22. DeClerck K and Elble RC: The role of hypoxia and acidosis in promoting metastasis and resistance to chemotherapy. Front Biosci (Landmark Ed) 15: 213-225, 2010.

23. Moldoveanu T, Follis AV, Kriwacki RW and Green DR: Many players in BCL-2 family affairs. Trends Biochem Sci 39: 101-111, 2014.

24. Snigdha S, Smith ED, Prieto GA and Cotman CW: Caspase-3 activation as a bifurcation point between plasticity and cell death. Neurosci Bull 28: 14-24, 2012.

25. Yamaguchi M: The anti-apoptotic effect of regucalcin is mediated through multisignaling pathways. Apoptosis 18: 1145-1153, 2013 